\title{
Power System Modeling of 20\% Wind-Generated Electricity by 2030
}

Maureen Hand and Nate Blair, National Renewable Energy Laboratory Mark Bolinger and Ryan Wiser, Lawrence Berkeley National Laboratory Richard O'Connell, Black and Veatch Tracy Hern and Bart Miller, Western Resources Advocates

\author{
Presented at IEEE Power and Energy Society \\ 2008 General Meeting held July 20-24, 2008 \\ Pittsburgh, PA \\ NREL/PR-500-44331 \\ NREL is operated by Midwest Research Institute . Battelle
}




\section{The Analytic Challenge:}

- Create technically and economically feasible scenario that estimates:

- Wind capacity in 2030 to produce $20 \%$ of projected electricity demand

- Geographic distribution of wind capacity

- Transmission system expansion requirements

- Direct electric system cost

- Portfolio of national generation technologies in 2030

- Potential natural gas price reduction

- Financial risk of future carbon regulation and avoided carbon emission

- Reduced water consumption 


\section{WinDS Model \\ (Wind Deployment Systems Model)}

A multi-regional, multi-time-period model of generation capacity and transmission infrastructure expansion in the U.S. electric sector.

Designed to estimate market potential of wind energy in the U.S. for the next $20-50$ years under different technology development and policy scenarios.

www.nrel.gov/analysis/winds 


\section{Approach}

- Use NREL's Wind Energy Deployment System (WinDS) generation capacity and transmission infrastructure expansion model

- Prescribe annual energy generation from wind technology up to $20 \%$ by 2030

- Assume future cost and performance for conventional and wind generation technologies

- Assume electric grid operation and expansion costs

- Select the nationally cost-optimized use of wind resource to meet annual energy production requirements using WinDS

- Optimizes use of different quality wind resources (Class $3-7$ ) in relation to load centers

- Optimizes use of existing vs. new transmission lines

- Optimizes relative cost of land-based and offshore wind technology in relation to load centers

- Optimizes balance of generation technologies without any assumption of future policy changes (e.g. no carbon mitigation policy) 


\section{WinDS Regions and Wind Resource}
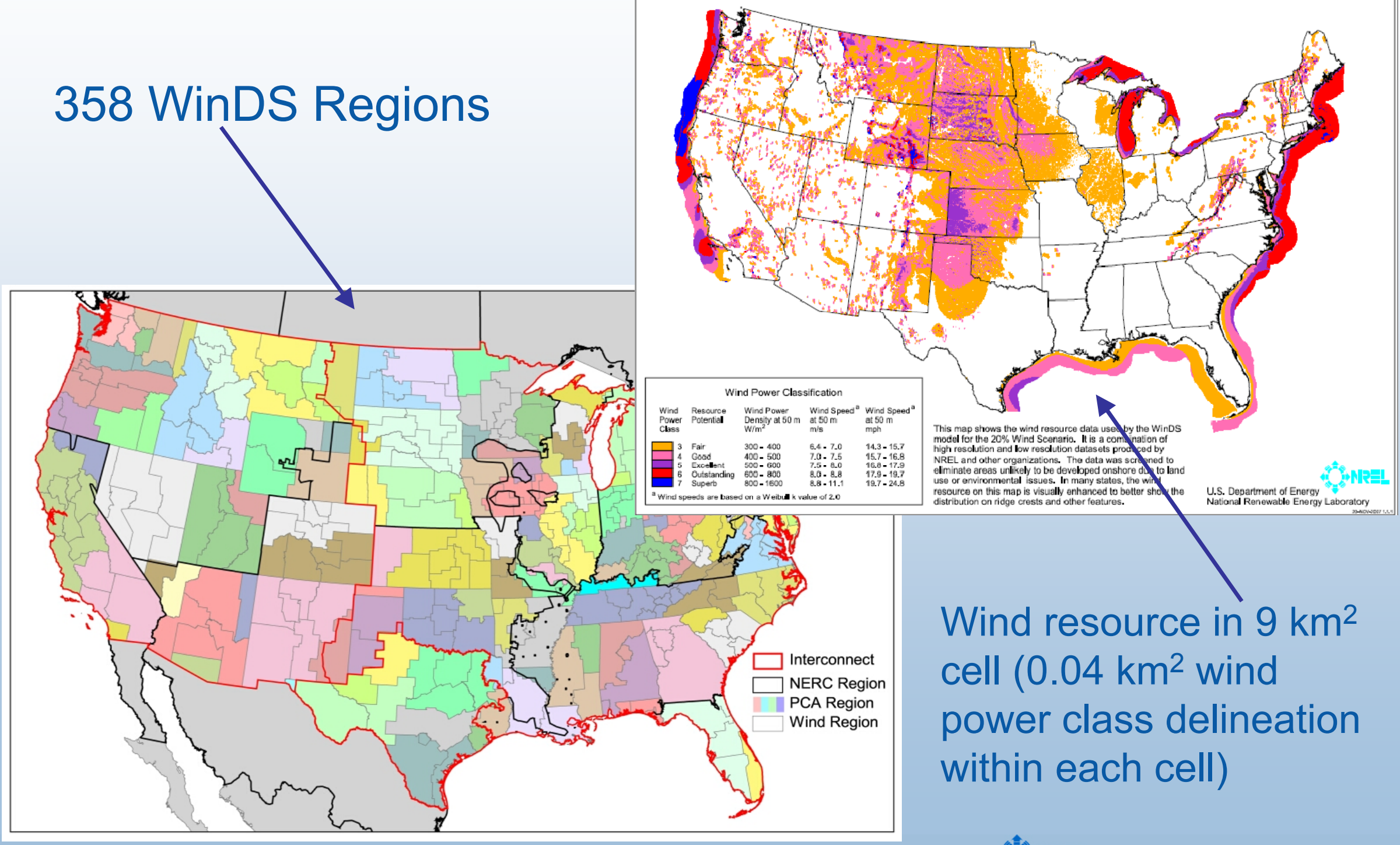

Wind resource in $9 \mathrm{~km}^{2}$ cell $\left(0.04 \mathrm{~km}^{2}\right.$ wind power class delineation within each cell) 


\section{Wind Energy Supply Curve}

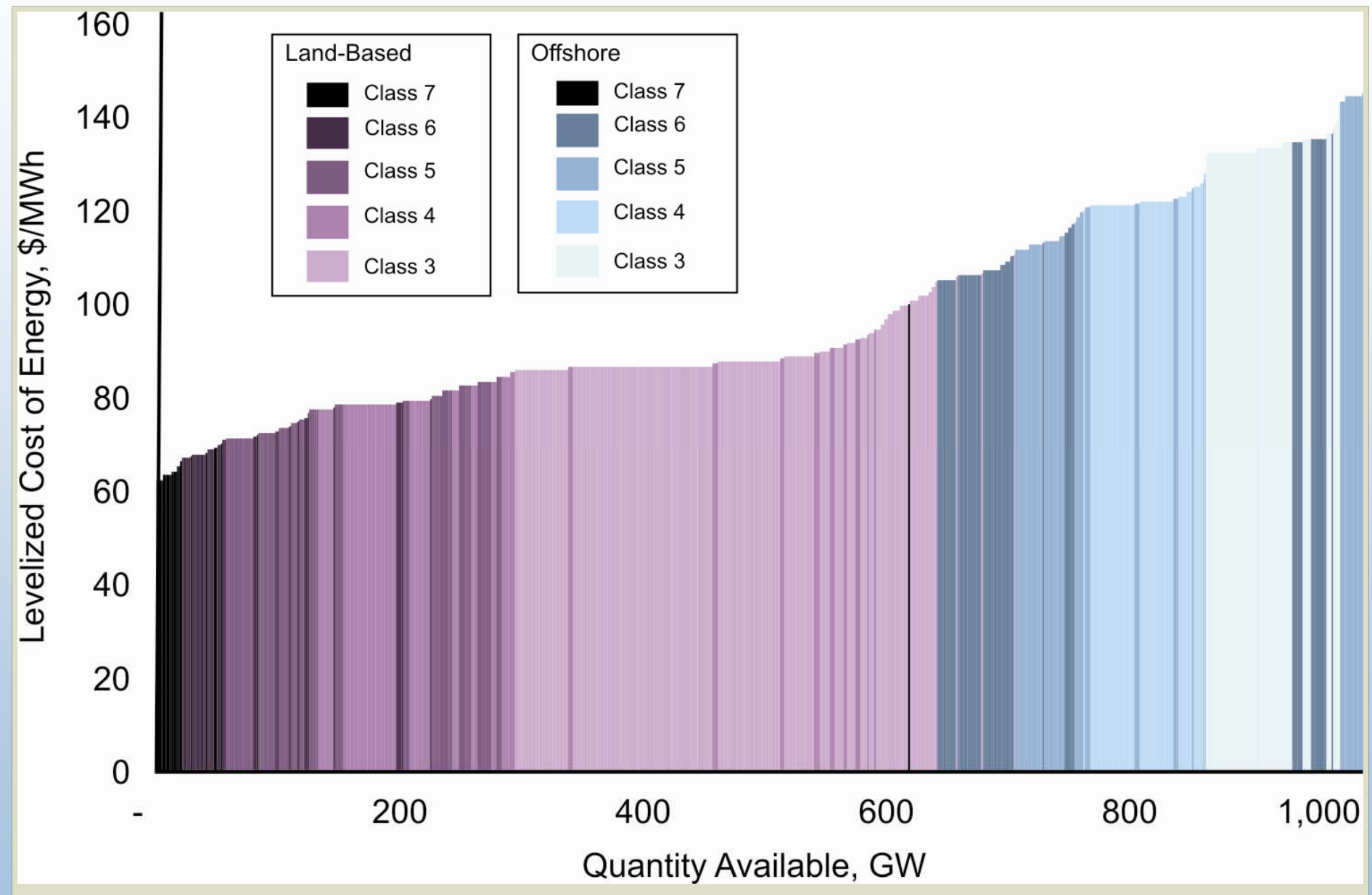

Excludes PTC, includes transmission costs to access $10 \%$ existing electric transmission capacity within $\mathbf{5 0 0}$ miles of wind resource. 


\section{$20 \%$ Wind Scenario}

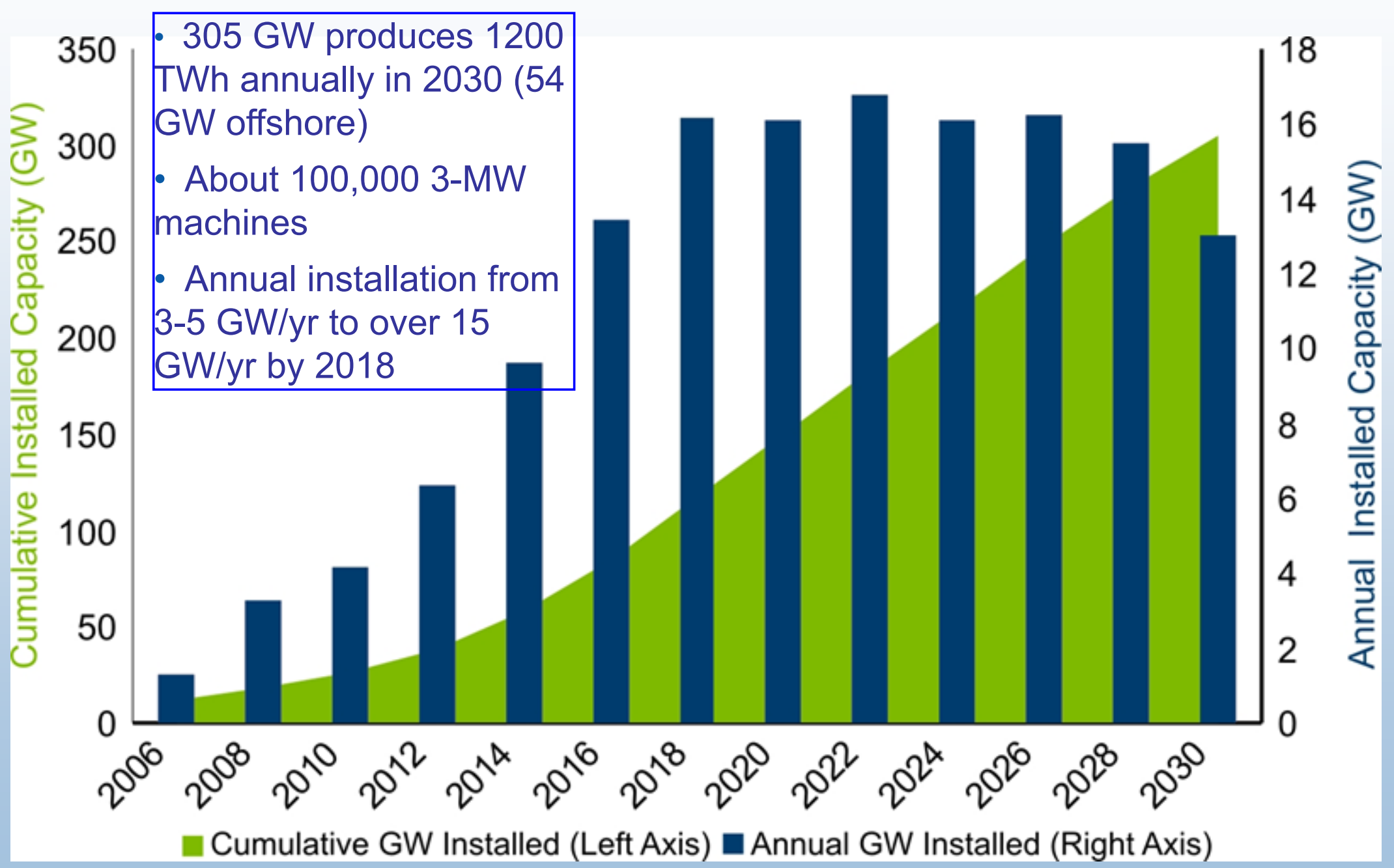




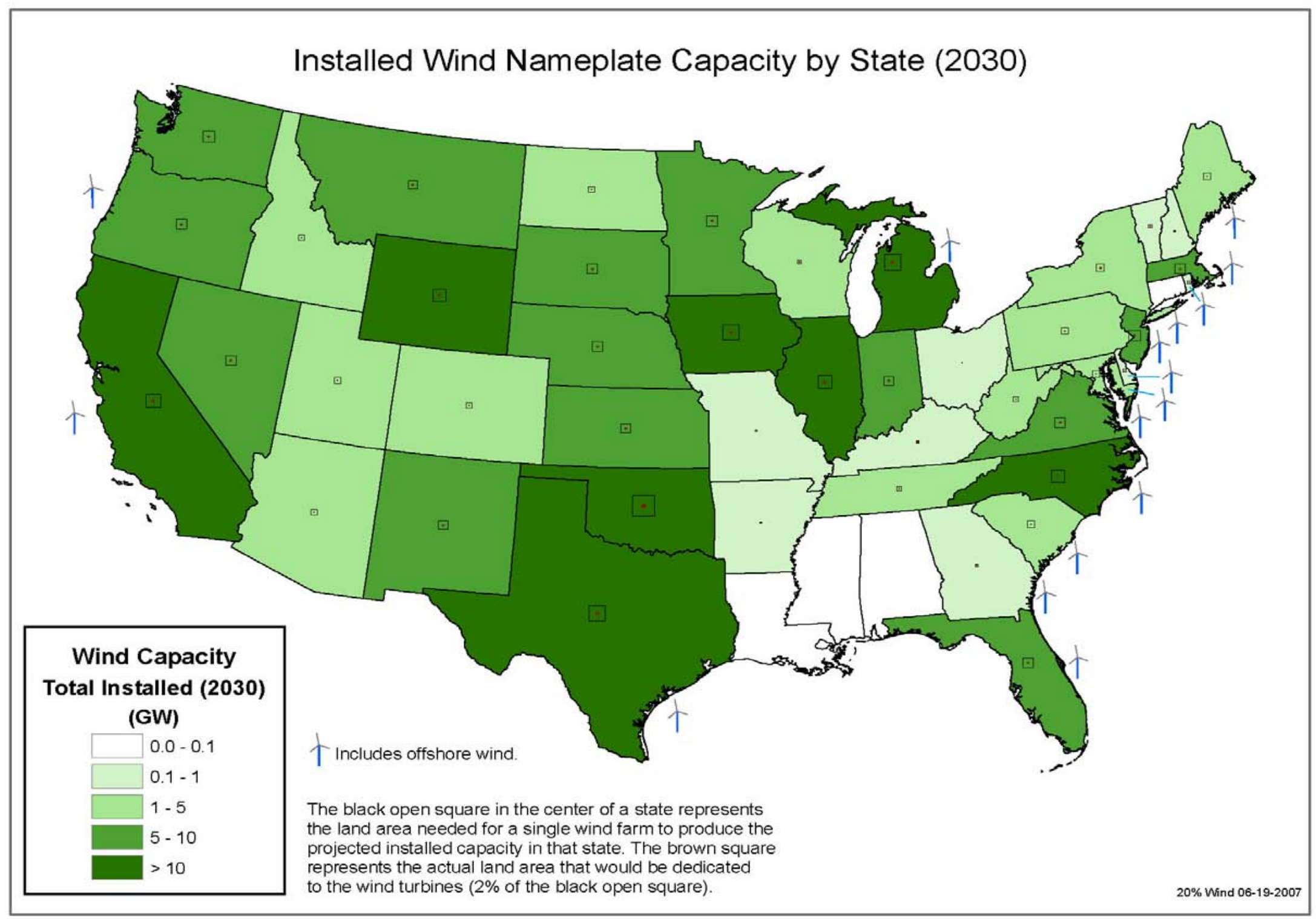




\section{Generation Technologies in 2030 with and without $20 \%$ Wind}
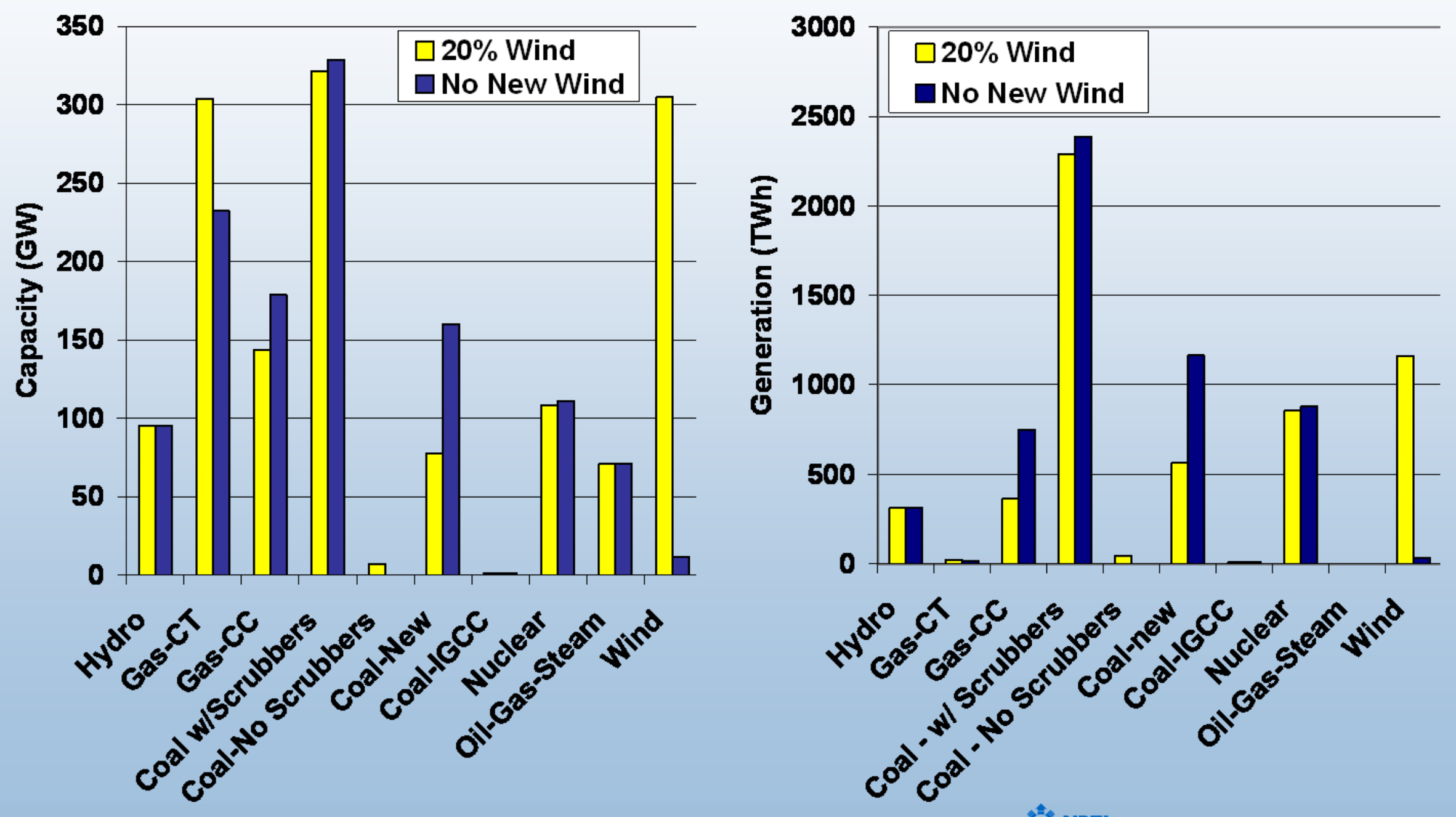

NRE- National Renewable Energy Laboratory 


\section{Generation Portfolios}
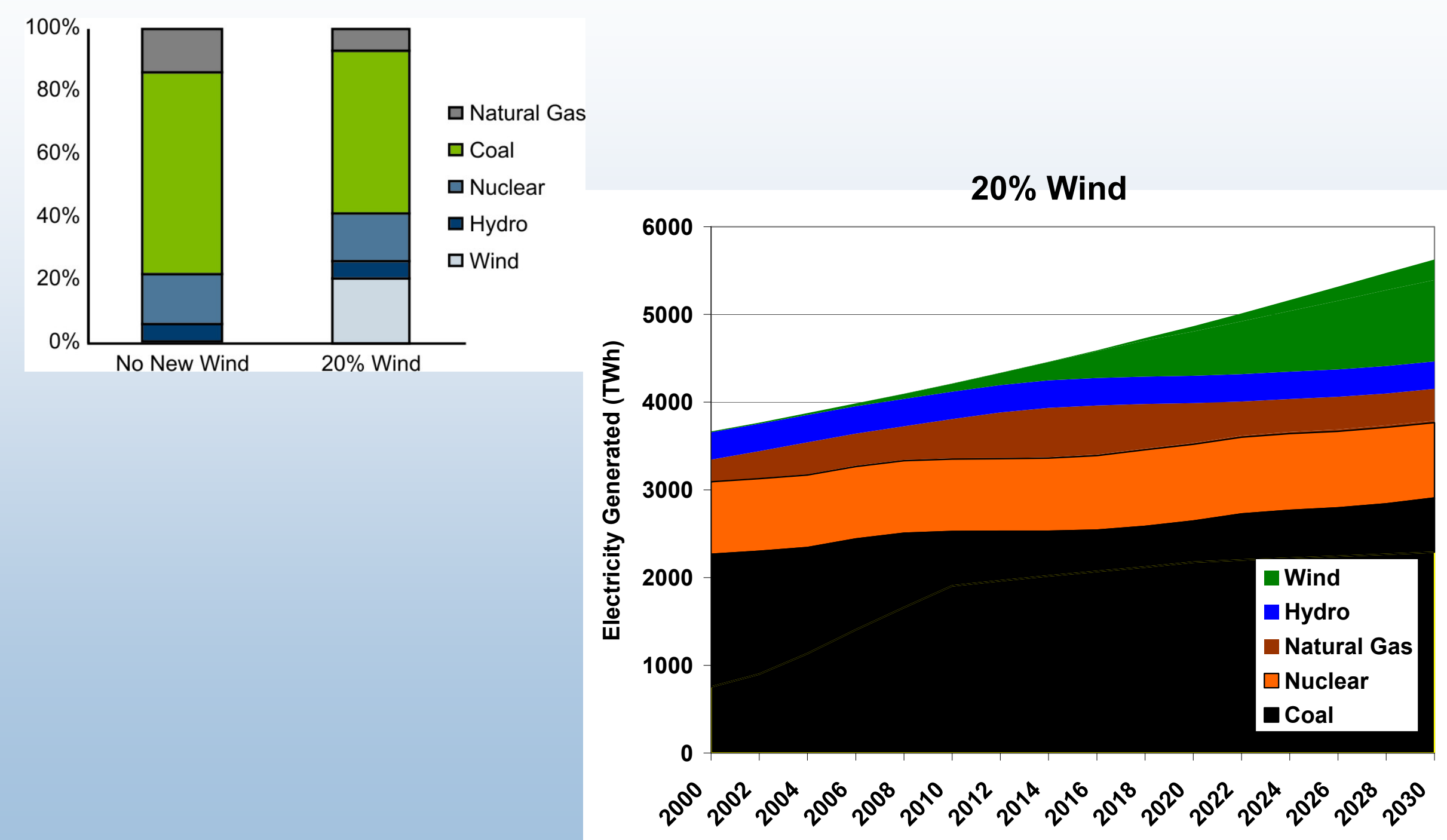


\section{Coal and Natural Gas Fuel Savings}

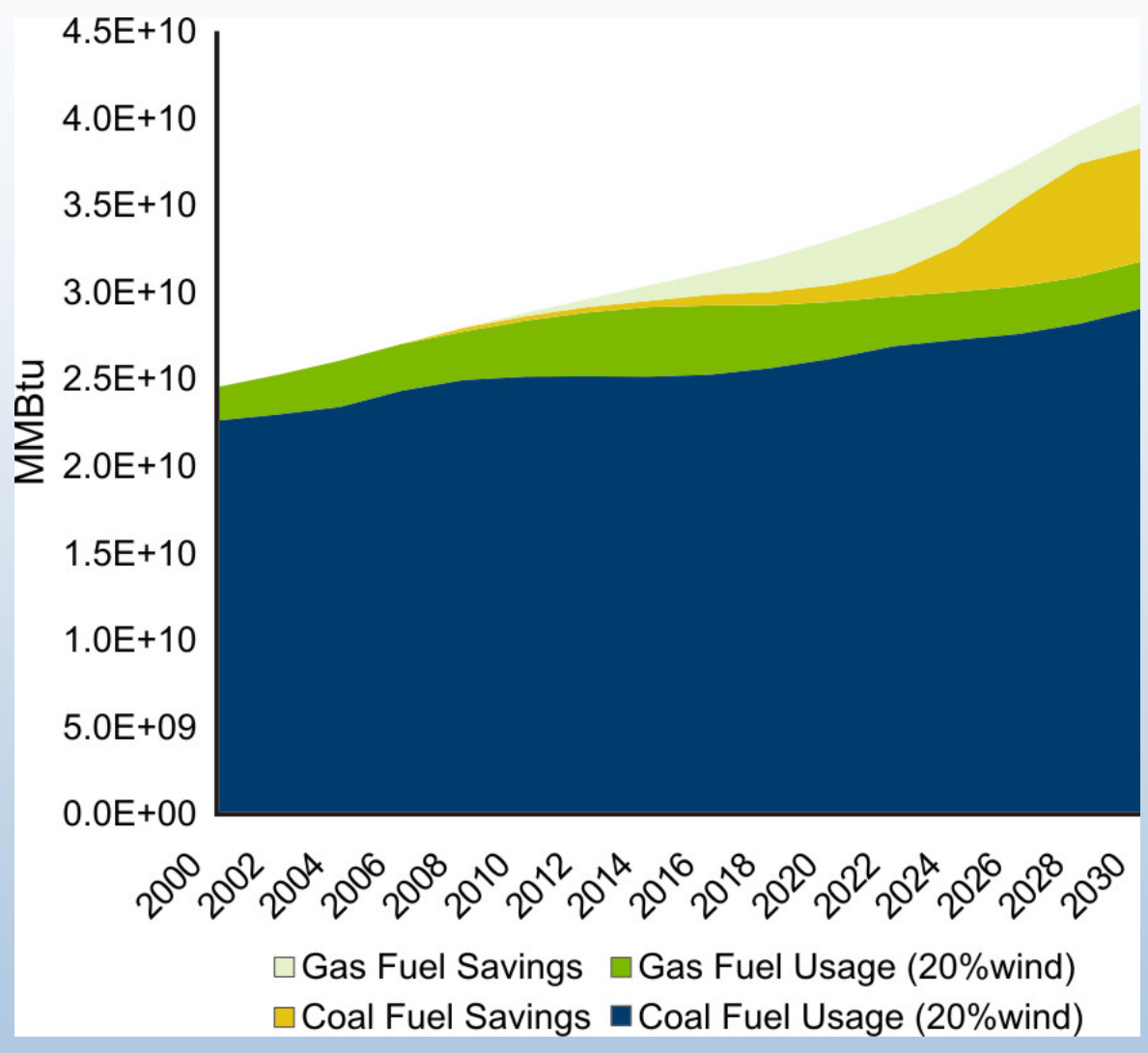

Electricity Sector Fuel Usage

\begin{tabular}{|c|c|c|c|}
\hline $\begin{array}{c}\text { \% Reduction in } \\
\text { Natural Gas } \\
\text { Consumption }\end{array}$ & $\begin{array}{c}\text { Natural Gas Price } \\
\text { Reduction in 2030 } \\
(2006 \$ / M M B t u)\end{array}$ & $\begin{array}{c}\text { Present Value Benefits } \\
\text { (billion 2006\$) }\end{array}$ & $\begin{array}{c}\text { Levelized Benefit of } \\
\text { Wind (2006\$/MWh) }\end{array}$ \\
\hline $11 \%$ & $0.6-1.5$ & $86-214$ & $16.6-41.6$ \\
\hline
\end{tabular}




\section{Avoided Carbon Dioxide Emissions}

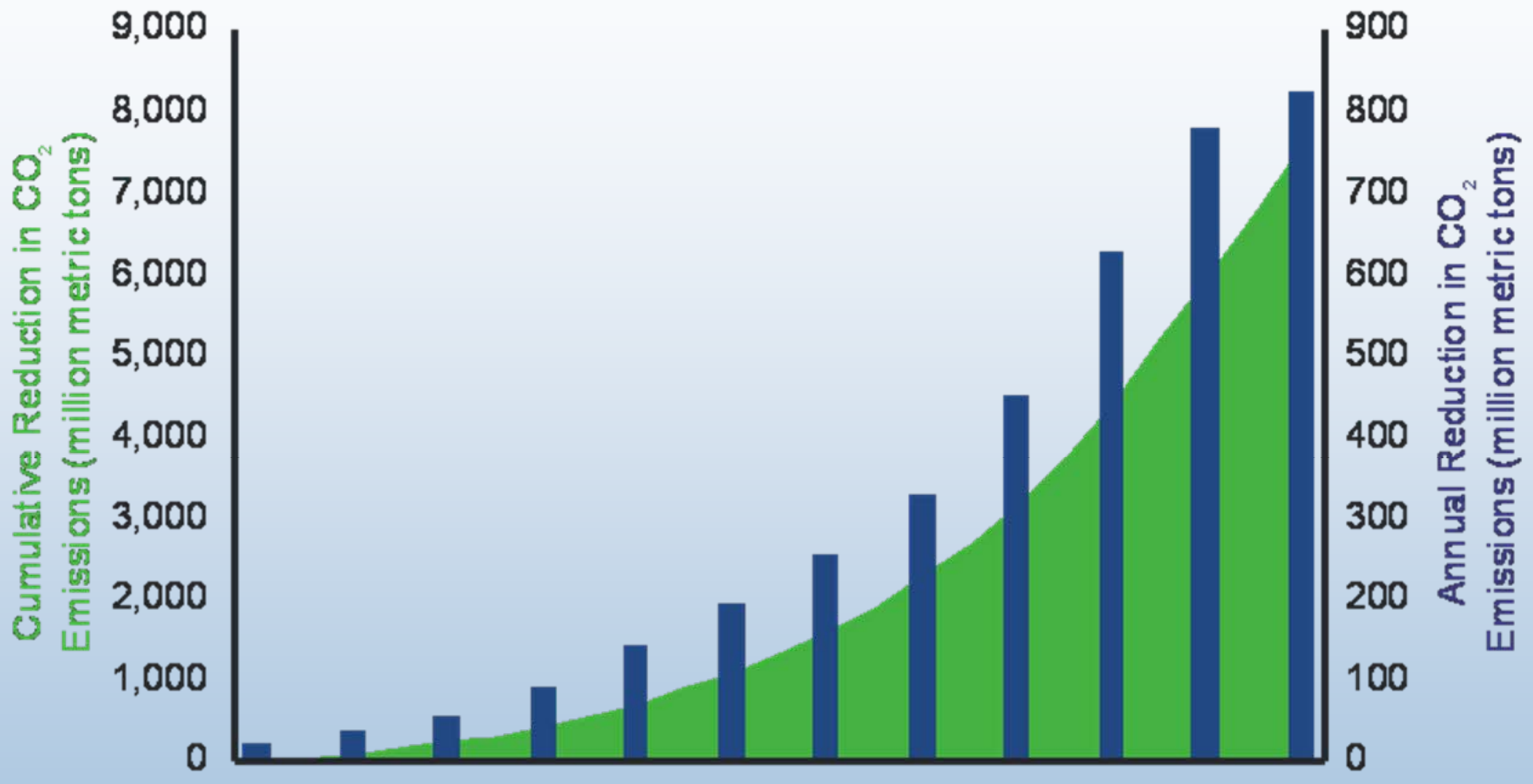

200820102012201420162018202020222024202620282030

- Cumulative Reductions (Left Axis) aAnnual Reductions (Right Axis) Valued from $\$ 50$ - \$145 billion in Net Present Value 


\section{Water Savings}

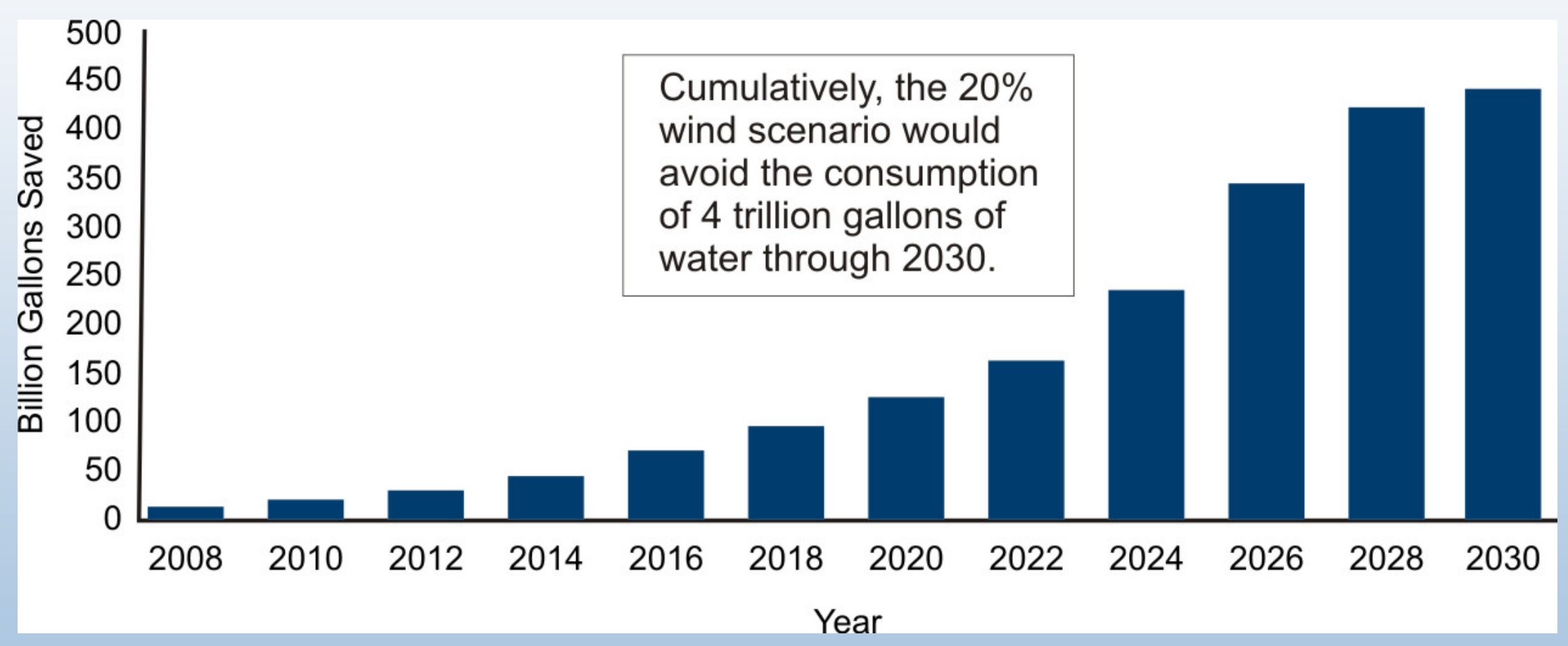




\section{Direct Electric System Cost}

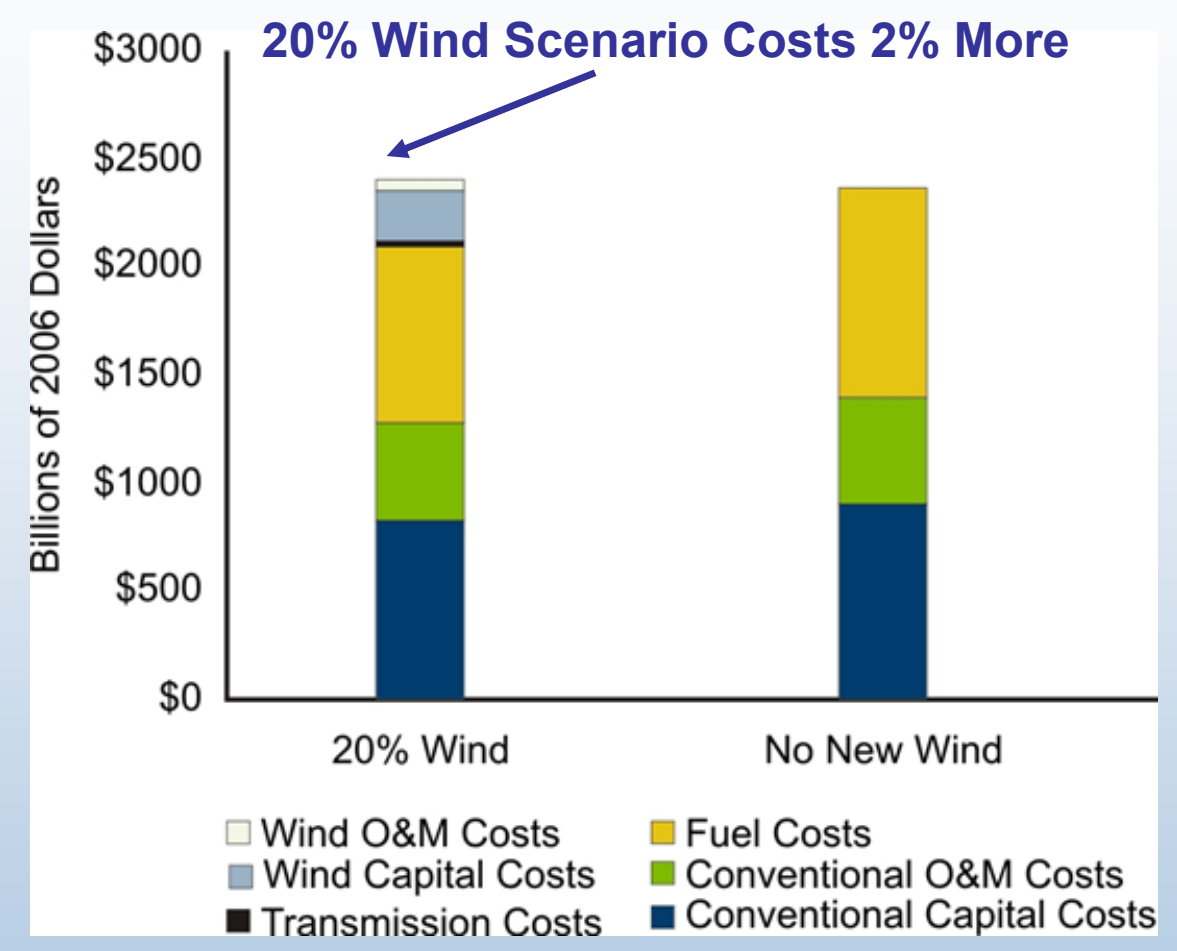

\begin{tabular}{|c|c|c|c|}
\hline $\begin{array}{c}\text { Present Value } \\
\text { Direct Costs } \\
\text { (billion } \$ 2006)\end{array}$ & $\begin{array}{c}\text { Average Incremental } \\
\text { Levelized } \\
\text { Cost of Wind } \\
(2006 \$ / M W h-W i n d)\end{array}$ & $\begin{array}{c}\text { Average Incremental } \\
\text { Levelized } \\
\text { Rate Impact } \\
(2006 \$ / M W h-T o t a l)\end{array}$ & $\begin{array}{c}\text { Impact on Average } \\
\text { Household Customer } \\
(2006 \$ / m o n t h)\end{array}$ \\
\hline$\$ 43$ billion & $\$ 8.6 / \mathrm{MWh}$ & $\$ 0.6 / \mathrm{MWh}$ & $\$ 0.5 /$ month \\
\hline
\end{tabular}




\section{Conclusions}

- Providing $20 \%$ of U.S. electricity from wind by 2030 is technically feasible

- Wind capacity in 46 states

- Transmission expansion required

- Benefits include (but not limited to) reduced

- Carbon emission and risk of financial consequences of future carbon regulation

- Natural gas prices

- Water consumption

- Incremental cost of wind technology modest compared to planned electric sector investment 


\section{Website (documentation and results) at: http://www.nrel.gov/analysis/winds}

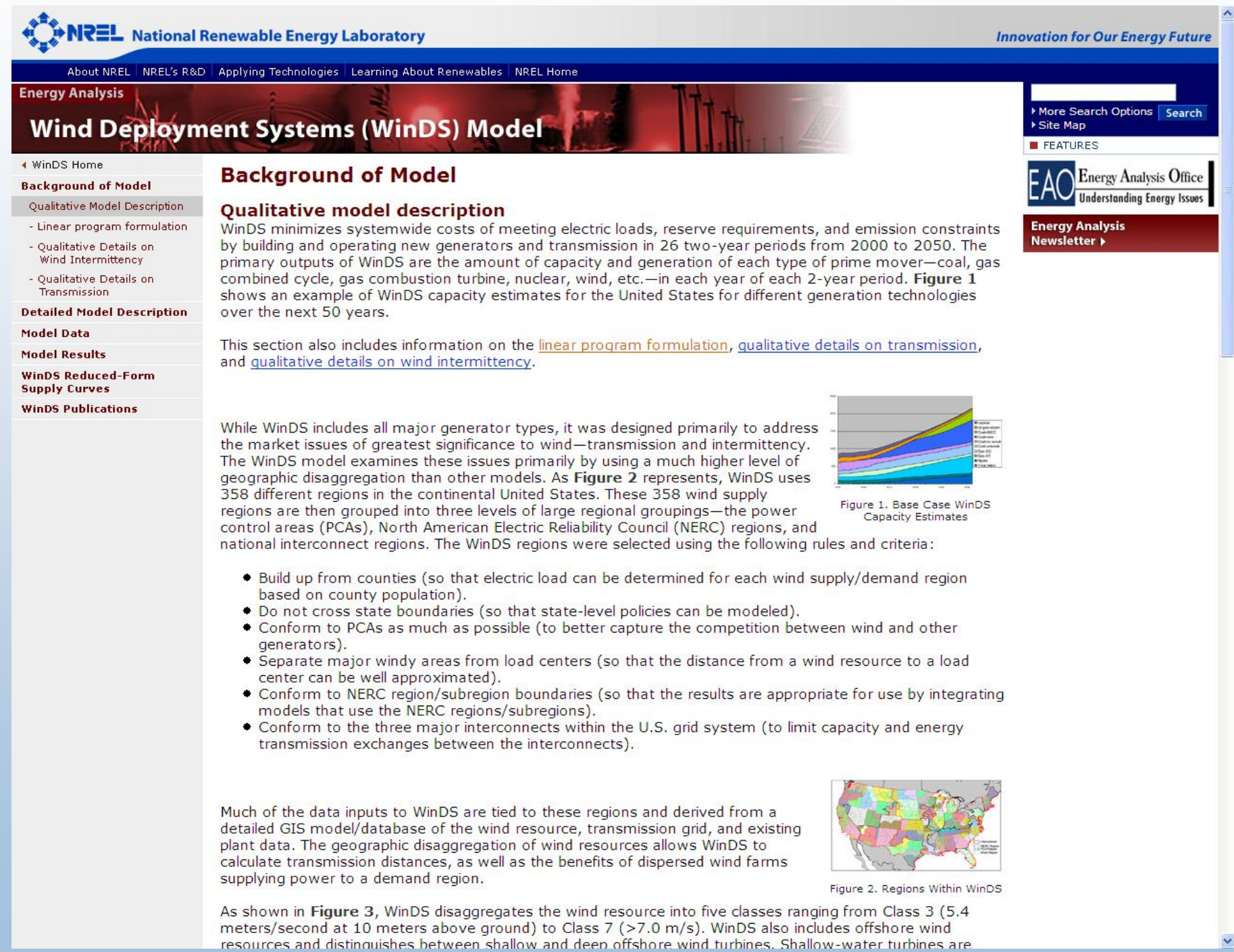

